Supplementary Information for:

\title{
Integrating social, economic, and environmental risk into flood management of aging dam infrastructure by combining cost-benefit and multi-criteria decision analyses
}

\author{
C.V. Castro, P.E. ${ }^{1}$ and H. S. Rifai, Ph.D., P.E. ${ }^{1}$ \\ Civil and Environmental Engineering, University of Houston
}

\section{Contents of this file:}

Supplementary Tables

SI Table S1 - HEC-HMS Subbasin Parameters for Addicks Watershed

SI Table S2 - HEC-HMS Subbasin Parameters for Buffalo Bayou Watershed

SI Table S3 - HEC-HMS Peak Values to HEC-RAS Flow Data

Supplementary Figures

SI Figure S1 - HEC-HMS Schematic for Addicks Watershed

SI Figure S2 - HEC-HMS Schematic for Buffalo Bayou Watershed

SI Figure S3 - HEC-RAS Schematic for Addicks Watershed

SI Figure S4 - NOAA Rainfall for Hurricane Harvey

SI Figure S5 - HEC-HMS Diversion and Discharge Charts for Cross-basin Overflow

\section{Supplementary Tables}

Supplementary Table S1: Parameter values for Addicks HEC-HMS subbasins using SCS Curve Number loss methodology. Values in ()$^{\dagger}$ indicate changes to the curve number and percentage of impervious coverage in each subbasin for Alternative A5 - Proposed Buyouts along the Buffalo Bayou.

\begin{tabular}{ccc|ccc}
\hline Subbasin & $\begin{array}{c}\text { Curve Number } \\
\text { (buyouts) }^{\dagger}\end{array}$ & $\begin{array}{c}\text { \% Impervious } \\
\text { (buyouts) }^{\dagger}\end{array}$ & Subbasin & $\begin{array}{c}\text { Curve Number } \\
\text { (buyouts) }^{\dagger}\end{array}$ & $\begin{array}{c}\text { \% Impervious } \\
\text { (buyouts }^{\dagger}\end{array}$ \\
\hline U101A & 56.08 & 14.99 & U106A & 57.18 & 46.93 \\
U101B & 55.91 & 17.62 & U106B & 57.67 & 45.87 \\
U101C & 55.76 & 17.83 & U106C & 57.42 & 52.39 \\
U101D & 56.23 & 36.28 & U106D & $58.13(51.63)$ & $52.54(46.66)$ \\
U101E & 55.79 & 35.24 & U120A & 56.22 & 44.87 \\
U101F & 57.65 & 52.33 & U129A & 56.15 & 8.40 \\
U101G & 57.89 & 52.94 & U129B & 57.06 & 39.85 \\
U101H & $58.12(55.12)$ & $54.45(51.64)$ & U129C & 57.47 & 50.24 \\
U101I & $58.81(54.77)$ & $40.36(37.59)$ & U129D & 57.56 & 54.18 \\
U102A & 56.28 & 18.42 & U129E & $57.94(52.01)$ & $58.67(52.67)$ \\
U102B & 55.41 & 20.87 & U129F & $60.33(57.81)$ & $57.13(47.07)$ \\
U102C & 56.60 & 45.70 & W167C & 63.13 & 30.19 \\
U102D & 58.30 & 43.11 & W167D & 57.01 & 42.38 \\
U102E & $58.31(57.44)$ & $44.37(43.71)$ & W167E & 59.21 & 56.04 \\
U102F & $59.49(58.43)$ & $31.49(30.93)$ & W167F & 59.64 & 58.78 \\
\hline
\end{tabular}




\section{Hydrology and Earth System Sciences}

Supplementary Table S2: Parameter values for Buffalo Bayou HEC-HMS subbasins using Green \& Ampt loss methodology. Values in ()$^{\dagger}$ indicate changes to the percentage of impervious coverage in each subbasin for Alternative A5 - Proposed Buyouts along the Buffalo Bayou.

\begin{tabular}{|c|c|c|c|c|c|}
\hline Subbasin & $\begin{array}{c}\text { Initial } \\
\text { Content }\end{array}$ & $\begin{array}{c}\text { Saturated } \\
\text { Content }\end{array}$ & $\begin{array}{l}\text { Suction } \\
\text { (in) }\end{array}$ & $\begin{array}{l}\text { Conductivity } \\
\text { (in/hr) }\end{array}$ & $\begin{array}{c}\text { \% Impervious } \\
\text { (buyouts) }^{\dagger}\end{array}$ \\
\hline W100A & 0.01 & 0.46 & 10.45 & 0.33 & 35 (34.98) \\
\hline W100B & 0.03 & 0.46 & 9.52 & 0.49 & 35 \\
\hline W100C & 0.01 & 0.48 & 8.3 & 0.37 & $45(44.82)$ \\
\hline W100D & 0.01 & 0.46 & 12.45 & 0.74 & $45(44.88)$ \\
\hline W100E & 0.01 & 0.46 & 12.45 & 0.74 & $45(44.88)$ \\
\hline W100F & 0.01 & 0.48 & 12.45 & 0.81 & $45(44.92)$ \\
\hline W100G & 0.01 & 0.48 & 12.45 & 0.81 & 45 (44.77) \\
\hline W100H & 0.03 & 0.46 & 10.03 & 0.37 & $40(39.86)$ \\
\hline W100I & 0.03 & 0.46 & 10.03 & 0.37 & $45(44.88)$ \\
\hline W100J & 0.03 & 0.46 & 10.03 & 0.37 & $40(39.87)$ \\
\hline W100K & 0.03 & 0.46 & 10.03 & 0.37 & 45 (44.97) \\
\hline W100L & 0.03 & 0.46 & 10.03 & 0.37 & 45 (44.98) \\
\hline W100M & 0.03 & 0.46 & 10.03 & 0.37 & $40(39.98)$ \\
\hline W100N & 0.03 & 0.46 & 10.03 & 0.37 & 50 \\
\hline W1000 & 0.03 & 0.46 & 10.03 & 0.37 & 45 \\
\hline W129A & 0.03 & 0.46 & 10.03 & 0.37 & 50 \\
\hline W138A & 0.03 & 0.46 & 10.03 & 0.37 & 50 \\
\hline W139A & 0.03 & 0.46 & 10.03 & 0.37 & 50 \\
\hline W140A & 0.03 & 0.46 & 10.03 & 0.37 & 45.18 \\
\hline W140B & 0.03 & 0.46 & 10.03 & 0.37 & 45 \\
\hline W140C & 0.03 & 0.46 & 10.03 & 0.37 & 45 \\
\hline W140D & 0.03 & 0.46 & 10.03 & 0.37 & 45 \\
\hline W140E & 0.03 & 0.46 & 10.03 & 0.37 & 45 \\
\hline W141A & 0.03 & 0.46 & 10.03 & 0.37 & 45.23 \\
\hline W142A & 0.03 & 0.46 & 10.03 & 0.37 & 55 \\
\hline W145A & 0.03 & 0.46 & 10.03 & 0.37 & 55 \\
\hline W147A & 0.01 & 0.48 & 12.45 & 0.81 & 55 \\
\hline W151A & 0.01 & 0.48 & 12.45 & 0.81 & $50.4(50.32)$ \\
\hline W156A & 0.01 & 0.46 & 12.45 & 0.74 & 55 \\
\hline W156B & 0.01 & 0.46 & 12.45 & 0.74 & 55 \\
\hline W167A & 0.01 & 0.46 & 9.1 & 0.37 & 52.47 \\
\hline W167B & 0.01 & 0.46 & 9.1 & 0.37 & 45 \\
\hline W170A & 0.04 & 0.46 & 6.8 & 0.34 & 43.26 \\
\hline W190A & 0.05 & 0.46 & 3.31 & 0.41 & 4.57 \\
\hline W190B & 0.05 & 0.46 & 3.31 & 0.41 & 3.11 \\
\hline W190C & 0.05 & 0.46 & 3.31 & 0.41 & 16.43 \\
\hline
\end{tabular}




\section{Hydrology and Earth System Sciences}

Supplementary Table S3: Values used to convert the HEC-HMS peak flow outputs at select junction nodes for each Alternative (A1-A8) into steady-state flow data for corresponding HEC-RAS cross-sections (XS). (Top: Addicks Reservoir Watershed, Bottom: Buffalo Bayou Watershed).

PEAK FLOW (cfs)

\begin{tabular}{lrlrrrr}
\hline RAS River & RAS XS & HMS Junction & \multicolumn{1}{c}{ A1 } & \multicolumn{1}{c}{ A2 } & A3 & \multicolumn{1}{c}{ A4 } \\
\hline U100-00-00 & 89149.7 & U1000000_0747_J & 3205.2 & 3205.2 & 3205.2 & 3205.2 \\
U100-00-00 & 62234.7 & U1000000_0613_J & 5918.9 & 5918.9 & 5918.9 & 5918.9 \\
U100-00-00 & 41734.5 & U1000000_0408_J & 12090.8 & 12090.8 & 12090.8 & 12090.8 \\
U100-00-00 & 39144.7 & U1000000_0386_J & 12660.8 & 12660.8 & 12660.8 & 12660.8 \\
U100-00-00 & 28751.6 & U1000000_0288_J & 13795 & 13795 & 13771 & 13795 \\
U100-00-00 & 27102.1 & U1000000_0219_J & 22486.8 & 22486.8 & 22407.6 & 22486.8 \\
U100-00-00 & 19514.7 & U1000000_0152_J & 22995.6 & 22995.6 & 21961.7 & 22995.6 \\
U101-00-00 & 101835.6 & U1010000_0959_J & 22593.8 & 4000 & 22593.8 & 5648.4 \\
U101-00-00 & 82695.4 & U1010000_0828_J & 25035.7 & 3670.2 & 25035.7 & 8393.7 \\
U101-00-00 & 67829.7 & U1010000_0660_J & 27615.1 & 7072.7 & 27615.1 & 11456.5 \\
U101-00-00 & 49146.2 & U1010000_0484_J & 31481.7 & 11975.9 & 31481.7 & 16164.8 \\
U101-00-00 & 36113.6 & U1010000_0361_J & 33537.8 & 15216.7 & 33537.8 & 19233 \\
U101-00-00 & 33216.3 & U1010000_0306_J & 34623 & 16650 & 34613.9 & 20610.5 \\
U101-00-00 & 26243.5 & U1000000_9902_J & 72745.8 & 56300.5 & 72605 & 60625.8 \\
U102-00-00 & 77737.9 & U1020000_0777_J & 10048.3 & 4007 & 10048.3 & 4277.5 \\
U102-00-00 & 58715.1 & U1020000_0587_J & 12346.8 & 8820 & 12346.8 & 9101.4 \\
U102-00-00 & 43042.76 & U1020000_0427_J & 13335.2 & 10933.7 & 13335.2 & 11188.5 \\
U102-00-00 & 19755.1 & U1020000_0198_J & 14901.4 & 13660.9 & 14817.2 & 13911.1 \\
U106-00-00 & 32133.5 & U1060000_0300_J & 1504.7 & 1504.7 & 1504.7 & 1504.7 \\
U106-00-00 & 22728 & U1060000_0227_J & 4751 & 4751 & 4751 & 4751 \\
U106-00-00 & 17601.8 & U1060000_0176_J & 7219.4 & 7219.4 & 7219.4 & 7219.4 \\
U106-00-00 & 6400.5 & U1060000_0006_J & 10139.8 & 10139.8 & 9927.2 & 10139.8 \\
\hline
\end{tabular}

PEAK FLOW (cfs)

\begin{tabular}{lrlrrrrr}
\hline RAS River & \multicolumn{1}{c}{ RAS XS } & HMS Junction & \multicolumn{1}{c}{ A1 } & \multicolumn{1}{c}{ A5 } & \multicolumn{1}{c}{ A6 } & \multicolumn{1}{c}{ A7 } & \multicolumn{1}{c}{ A8 } \\
\hline W100-00-00 & 248647.7 & W1000000_2411_J & 6300 & 6300 & 3576.3 & 6300 & 3694.4 \\
W100-00-00 & 241079 & W1000000_0020_J & 13300 & 13300 & 3800 & 13300 & 6984.3 \\
W100-00-00 & 232681.7 & W1000000_2271_J & 16700 & 16700 & 4000 & 16700 & 8611.2 \\
W100-00-00 & 239993.6 & W1000000_2340_J & 16700 & 16700 & 6374.6 & 16700 & 6359.9 \\
W100-00-00 & 214669.3 & W1000000_2147_J & 19000 & 19000 & 11703.4 & 19000 & 11371.5 \\
W100-00-00 & 211631.3 & W1000000_2116_J & 19000 & 19000 & 12029.7 & 19000 & 11659.9 \\
W100-00-00 & 205679.6 & W1000000_2037_J & 19000 & 19000 & 12340.6 & 19000 & 11928.1 \\
W100-00-00 & 199440.6 & W1000000_1985_J & 19000 & 19000 & 13626.1 & 19000 & 13195.7 \\
W100-00-00 & 196182.3 & W1000000_1879_J & 17000 & 17000 & 13626.1 & 17000 & 14929.5 \\
W100-00-00 & 128104.6 & W1000000_1237_J & 24000 & 24000 & 13626.1 & 24000 & 27523.8 \\
W100-00-00 & 188903.7 & W1000000_1865_J & 24000 & 24000 & 15401.7 & 24000 & 14923.5 \\
W100-00-00 & 175675.6 & W1000000_1757_J & 24000 & 24000 & 17501.3 & 24000 & 17028 \\
W100-00-00 & 166558.2 & W1000000_1663_J & 24000 & 24000 & 24028 & 24000 & 17526.3 \\
W100-00-00 & 162811.9 & W1000000_1646_J & 24000 & 24000 & 24852 & 24000 & 23320.7 \\
\hline
\end{tabular}




\section{Supplementary Figures}

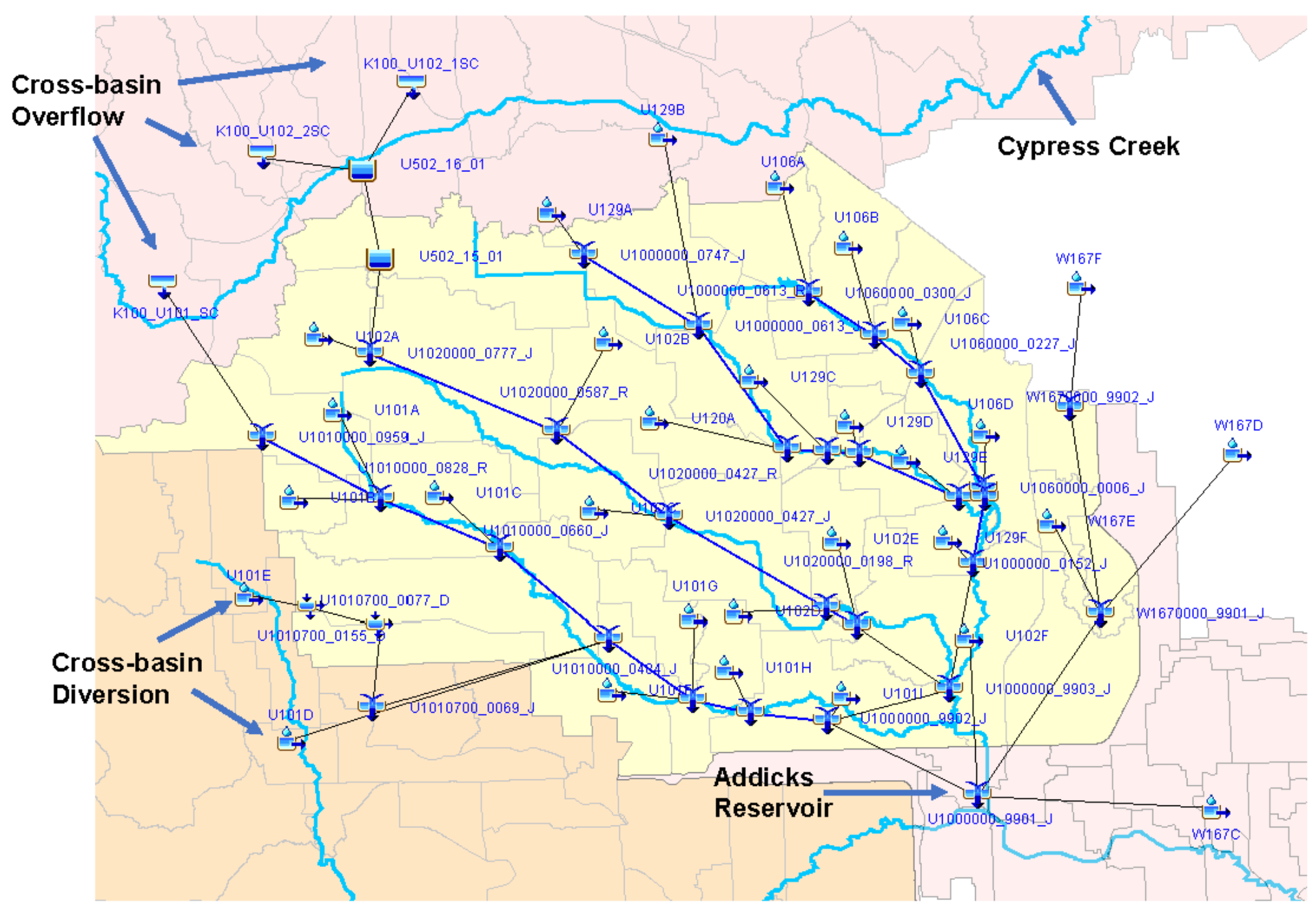

Supplementary Figure S1: HEC-HMS schematic for Addicks watershed model, showcasing how the cross-basin overflow from Cypress Creek was integrated into model through diversion nodes. 


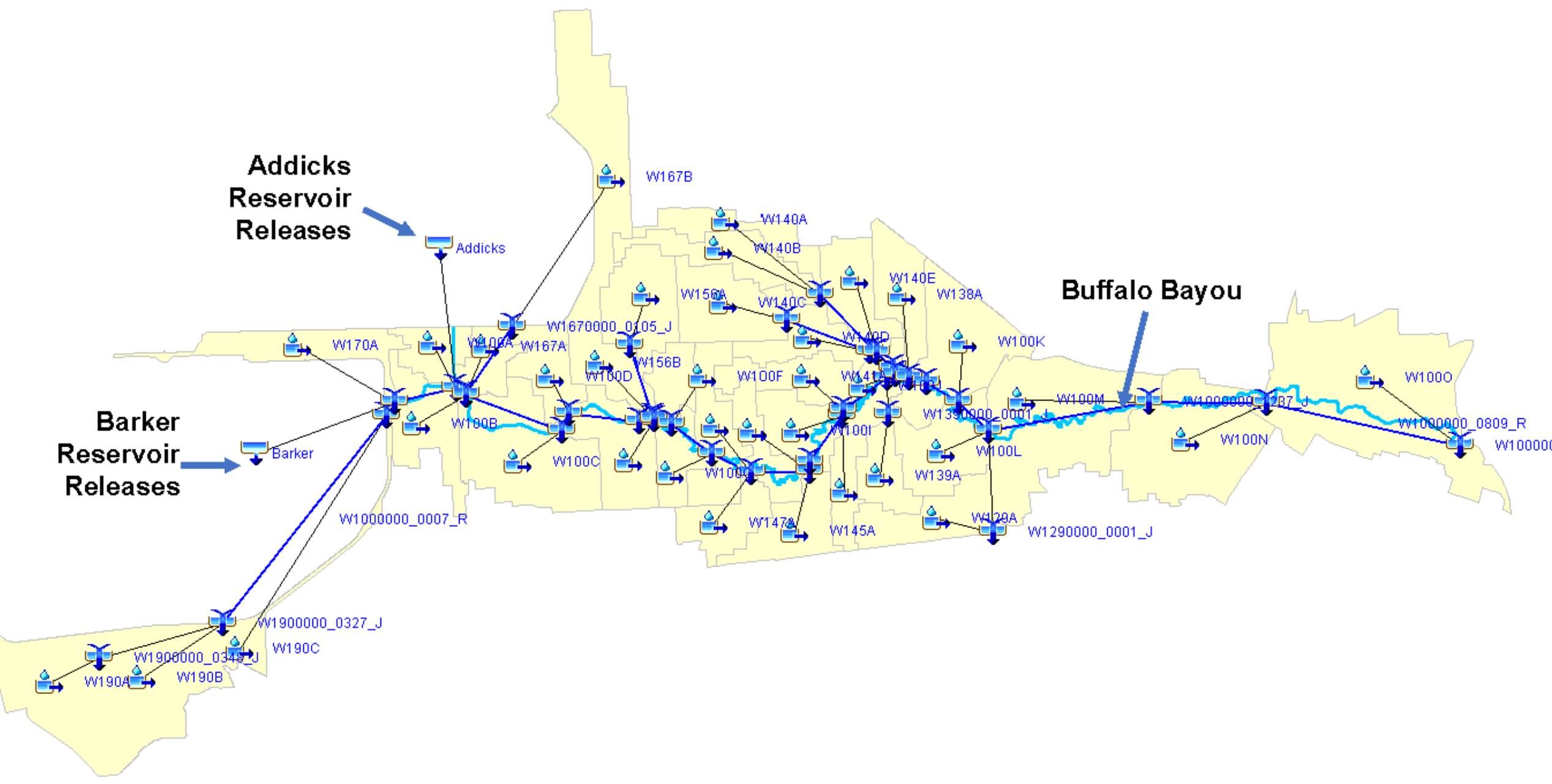

Supplementary Figure S2: HEC-HMS schematic for Buffalo Bayou watershed model, showcasing how the Addicks and Barker reservoir releases were integrated into the Buffalo Bayou watershed model through source discharge gages. 


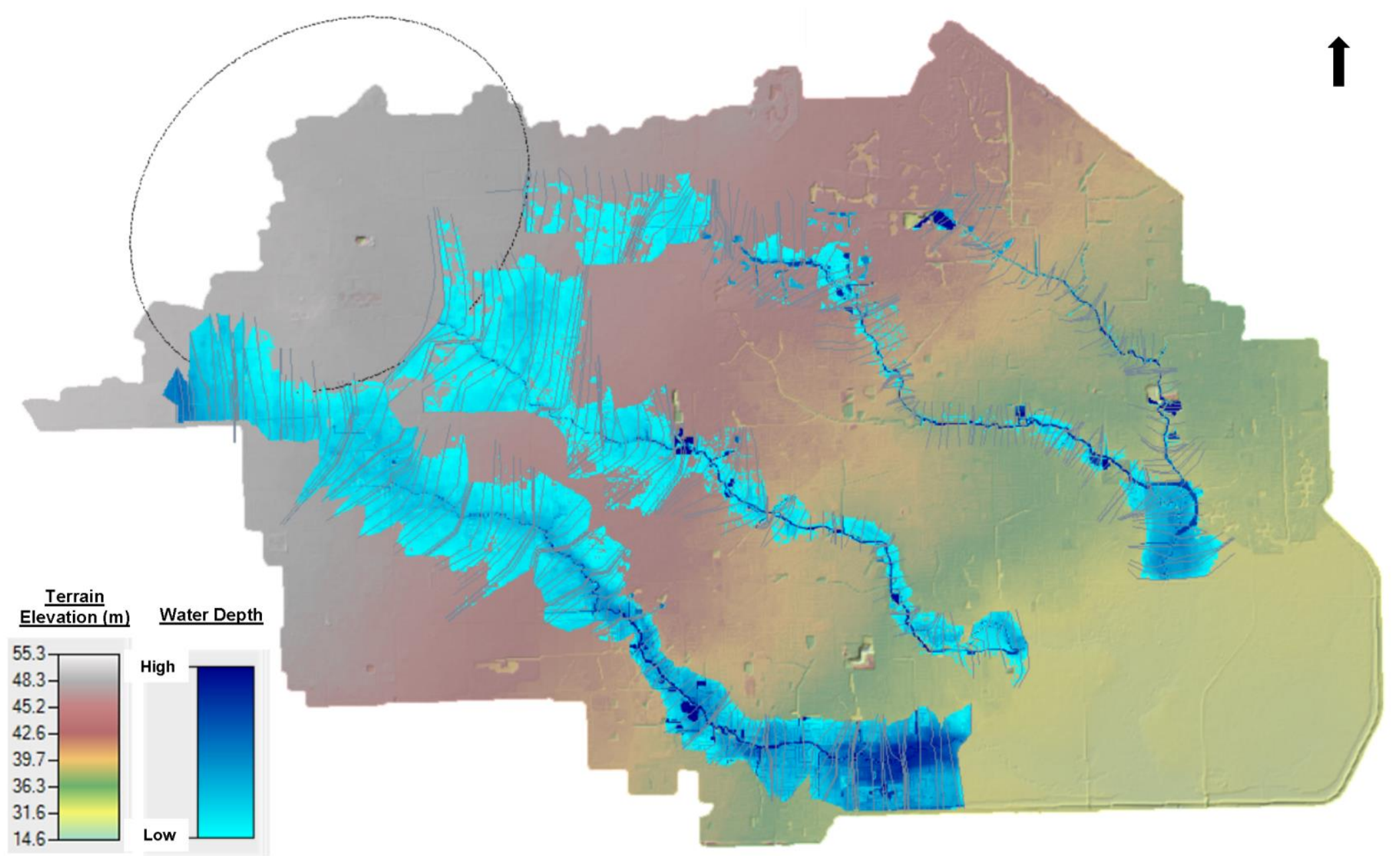

Supplementary Figure S3: HEC-RAS schematic for Addicks watershed model, showcasing how the various streams within the basin were combined within a single model to estimate flood inundation bounds and water depth under Hurricane Harvey conditions. 


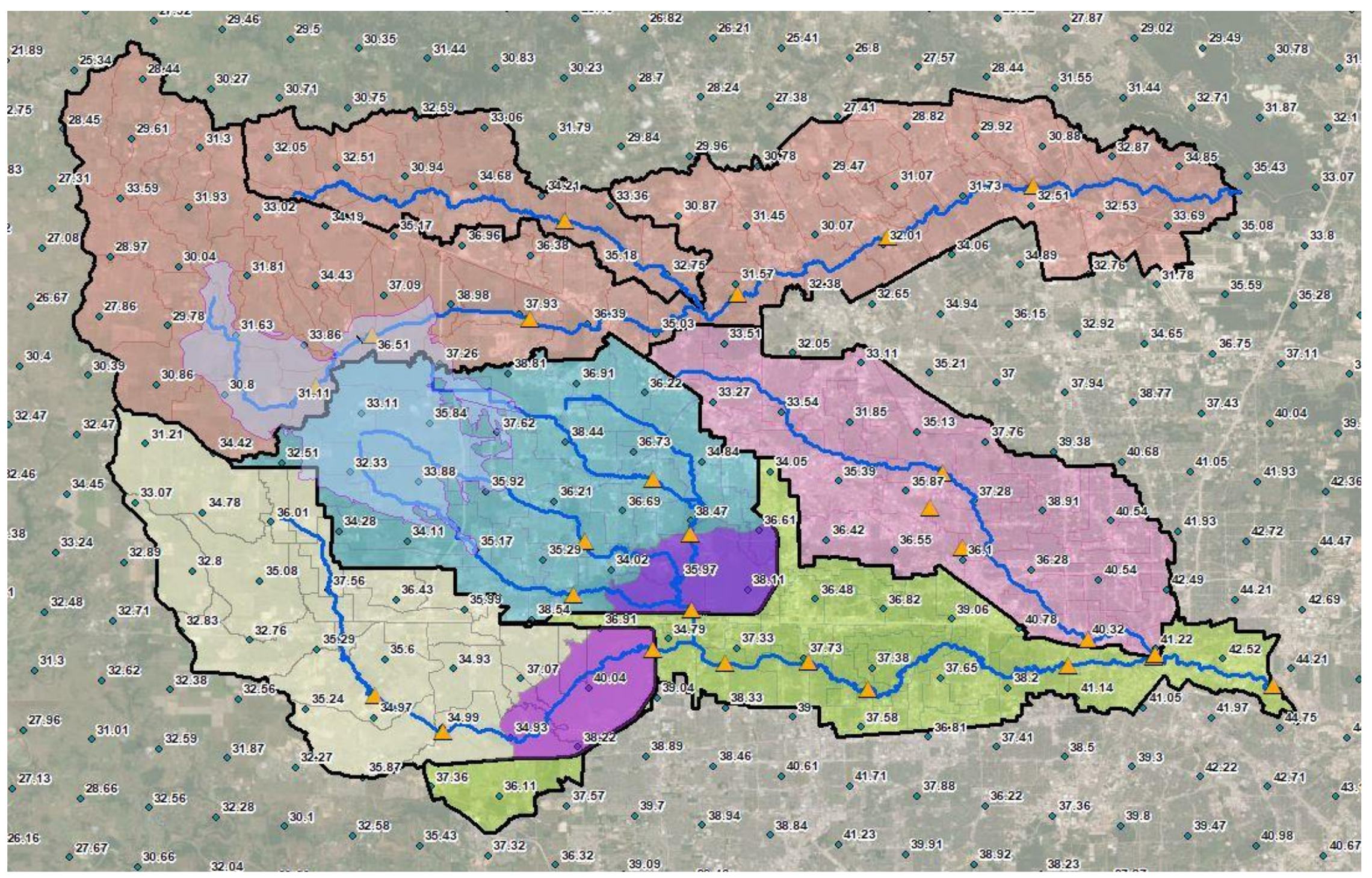

Supplementary Figure S4: Gridded rainfall maximum values for Hurricane Harvey conditions in the ABRS inter-connected watershed system, from National Oceanic Atmospheric Administration (NOAA, 2017a) for August 24, 2017 21:00 to August 29, 2017 23:00. 

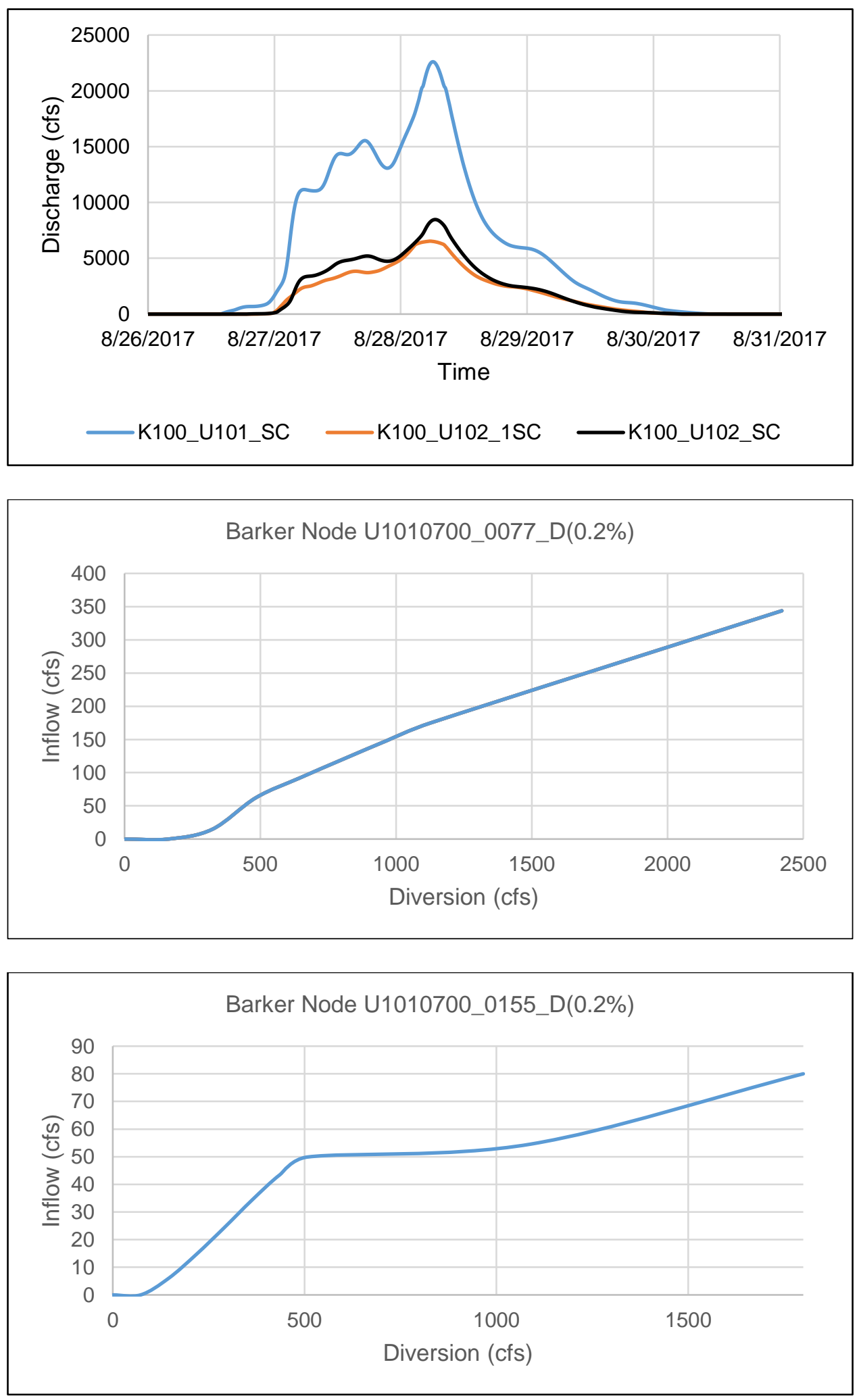

Supplementary Figure S5: Discharge and diversion node values used to link the Cypress Creek overflow conditions with the Addicks watershed HEC-HMS model. 